\title{
Direct imaging of current-induced domain wall motion in CoFeB structures
}

\author{
L. Heyne, M. Kläui, ${ }^{a)}$ D. Backes, ${ }^{\text {b) }}$ P. Möhrke, T. A. Moore, J. G. Kimling, O. Boulle, and \\ U. Rüdiger \\ Fachbereich Physik, Universität Konstanz, Universitätsstr. 10, 78457 Konstanz, Germany \\ L. J. Heyderman \\ Laboratory for Micro- and Nanotechnology, Paul Scherrer Institut, CH-5232 Villigen PSI, Switzerland
}

A. Fraile Rodríguez and F. Nolting

Swiss Light Source, Paul Scherrer Institut, CH-5232 Villigen PSI, Switzerland

K. Kirsch and R. Mattheis

Institute of Photonic Technology (IPHT), Jena, P.O. Box 100239, D-07702 Jena, Germany

(Presented on 7 November 2007; received 12 September 2007; accepted 2 November 2007; published online 18 March 2008)

\begin{abstract}
By direct x-ray photoemission electron microscopy imaging, we probe current-induced domain wall motion in $20 \mathrm{~nm}$ thick $\mathrm{CoFeB}$ wires. We observe transverse walls for all wire widths up to $1500 \mathrm{~nm}$ as a consequence of the small saturation magnetization of the material. High critical current densities above $1 \times 10^{12} \mathrm{~A} / \mathrm{m}^{2}$ for wall displacement due to the spin transfer torque effect are found. The critical current densities $j_{c}$ increase further with decreasing wire width indicating that $j_{c}$ is governed by extrinsic pinning due to edge defects. In addition to wall displacements, we observe wall transformations to energetically favorable wall types due to heating. Owing to the high Curie temperature though, the sample temperature stays below the Curie temperature even for the highest current densities where structural damage sets in. () 2008 American Institute of Physics. [DOI: $10.1063 / 1.2836326]$
\end{abstract}

\section{INTRODUCTION}

Control and manipulation of magnetization by current injection has recently become a topic of great interest since, in addition to exciting fundamental physics, novel applications based on current-induced rather than the conventional field-induced switching have been proposed. ${ }^{1,2}$ In such devices, magnetization reversal occurs by current-induced domain wall motion (CIDM), which was theoretically predicted some time ago ${ }^{3,4}$ and has also been thoroughly studied by numerical simulations. ${ }^{5,6}$ Experimentally, such devices have been recently realized by a number of groups measuring critical current densities, domain wall displacements, and domain wall transformations using a range of techniques. ${ }^{7-11}$ One of the key problems at the moment is the high current density necessary for CIDM, which results in heating due to Ohmic losses that can induce random domain wall displacements and even the nucleation and annihilation of domain walls if the samples are heated above the Curie temperature. $^{12,13}$ To overcome this problem, different approaches have been proposed. If the critical current density is governed by the interplay between the adiabatic torque term and the anisotropy, ${ }^{14}$ the transverse anisotropy and the damping constant can be engineered to reduce the critical current density $j_{c} .{ }^{15}$ In the case of extrinsic pinning at defects such as edge irregularities and grain boundaries intrinsic to the material, the critical current density was found to scale with the critical fields for wall displacement, which manifests it-

\footnotetext{
${ }^{a)}$ Electronic mails: mathias.klaeui@uni-konstanz.de and mathias@klaeui.de

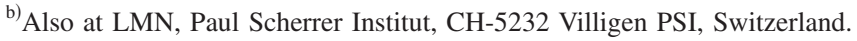

self for instance in an inverse scaling behavior of $j_{\text {crit }}$ versus the wire width ${ }^{16}$ as well as the critical field for field-induced displacement $H_{\text {crit }}{ }^{17}$

Smaller coercive fields than in the usually used Permalloy $\left(\mathrm{Ni}_{80} \mathrm{Fe}_{20}\right)$ have been measured for $\mathrm{CoFeB}$ and, recently, very low critical current densities have been observed for current-induced domain wall motion in multilayers made of $\mathrm{Co} / \mathrm{Cu} / \mathrm{CoFeB} .{ }^{15} \mathrm{CoFeB}$ that is grown without annealing is known to be amorphous and thus exhibits no grain boundaries where pinning could occur.

In this paper, we investigate current-induced domain wall motion in single layer $\mathrm{CoFeB}$ wires using nonintrusive $\mathrm{X}$-ray magnetic circular dichroism photoemission electron microscopy (XMCDPEEM). We image domain wall displacements and study wall transformations. We probe the behavior for current densities up to $5 \times 10^{12} \mathrm{~A} / \mathrm{m}^{2}$ and investigate the onset of structural damage at higher current densities.

\section{EXPERIMENTAL}

A topographic scanning electron microscopy (SEM) image of a set of zig-zag wires is presented in Fig. 1(a). The wires were fabricated by electron beam lithography and sputtering from a $\mathrm{CoFeB}$ target $\left(\mathrm{Co}_{66} \mathrm{Fe}_{22} \mathrm{~B}_{12}\right)$ without subsequent annealing, preserving the amorphous state. The thickness of the $\mathrm{CoFeB}$ is $20 \mathrm{~nm}$ with a $2 \mathrm{~nm}$ thick Ru capping layer, and the wire width varies between 220 and $1500 \mathrm{~nm}$. In a second step, $\mathrm{Au}$ contacts were added at the ends of the wires ${ }^{18}$ to allow for current injection using $25 \mu$ s long pulses with varying amplitude. One of the drawbacks of using 

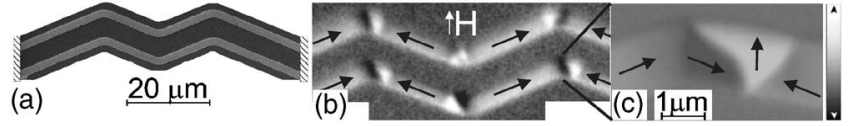

FIG. 1. (a) Topographic SEM image of $\mathrm{CoFeB}$ zig-zag wires $(20 \mathrm{~nm}$ thick and $1500 \mathrm{~nm}$ wide). The Au pads are the hatched areas at the wire ends. (b) XMCDPEEM image showing the corresponding magnetization configuration after applying an external magnetic field along the direction indicated by the white arrow and relaxing the field back to zero. Different shades of gray indicate the direction of the magnetic spins (see gray scale bar) and black arrows are used to visualize them. In (c), a high resolution image is shown of a transverse wall at one of the kinks.

$\mathrm{CoFeB}$ compared to $3 d$ metals or their alloys is the higher resistivity. ${ }^{19}$ In our wire samples the resistivity is about a factor 2.5 higher $(170 \mu \Omega \mathrm{cm})$ than for Permalloy wires $(70 \mu \Omega \mathrm{cm})$. This means that for identical geometries a voltage that is 2.5 times higher than that used for Permalloy is needed to obtain the same current density.

To image the magnetization, XMCDPEEM is used with the energy set to the $\mathrm{Fe}-\mathrm{L}_{3}$ absorption edge, ${ }^{11,20}$ after sputter removal of the capping layer.

\section{RESULTS AND DISCUSSION}

The sample was initialized by a strong in-plane magnetic field in the direction indicated by the white arrow in Fig. 1(b). The resulting magnetization configuration with the magnetization pointing in opposite directions in adjacent branches of the wires and transverse head-to-head and tailto-tail domain walls at the kinks is shown in Fig. 1(b). A high resolution image of a transverse head-to-head domain wall in a $1500 \mathrm{~nm}$ wide wire is shown in Fig. 1(c). For Permalloy wires with the same dimensions, we observe vortex walls after initialization, ${ }^{21}$ which means that compared to Permalloy the phase boundary between transverse and vortex walls is shifted to larger thicknesses and widths. This can be explained by the fact that the saturation magnetization of CoFeB $[0.75 \mathrm{~T}$ (Ref. 22)] is smaller than that of Permalloy $(>1 \mathrm{~T})$, which lowers the stray field energy, and thus makes transverse walls energetically more favorable. As expected, such transverse walls were also observed for all the other smaller wire widths investigated. The wall widths are similar to those we observe in Permalloy for similar geometries corroborating our earlier observation that for these geometries the wall widths are governed primarily by the geometry. ${ }^{23}$

When current is injected, different effects can occur. If the initial domain wall type observed is a metastable state that is separated from the lower energy wall type by an energy barrier, the Joule heating will allow this energy barrier to be overcome and the wall will transform to the lower energy wall type. ${ }^{21}$ For sufficiently high current densities, the wall will then be moved in the electron flow direction due to the spin transfer torque effect. In Fig. 2(a), a wire is shown before current injection, with a transverse wall located at the kink. We then gradually increase the current density in steps of $10^{11} \mathrm{~A} / \mathrm{m}^{2}$ until we observe a change in the magnetization configuration. We find no changes up to a current density of $1 \times 10^{12} \mathrm{~A} / \mathrm{m}^{2}$, at which value $25 \mu$ s pulse injections move the wall in the electron flow direction, as seen in Fig. 2(b). Furthermore the wall also transforms to a vortex wall. This
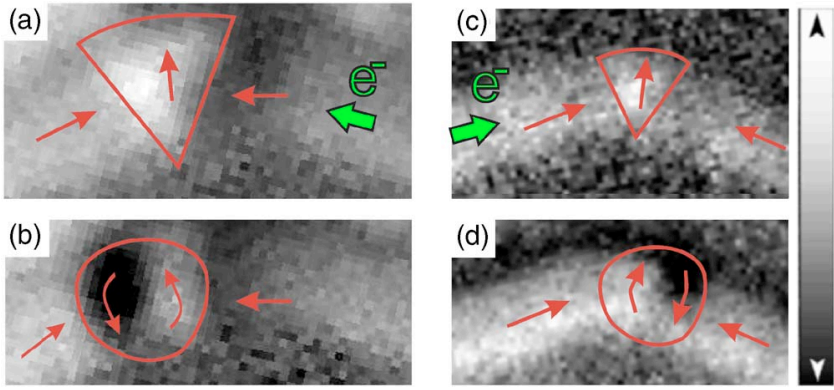

FIG. 2. (Color online) XMCDPEEM images demonstrating CIDM in $\mathrm{CoFeB}$. The original transverse wall in the $1500 \mathrm{~nm}$ wide wire (a) is displaced in the direction of the current flow (indicated by the arrow) after a pulse injection $\left(j=1 \times 10^{12} \mathrm{~A} / \mathrm{m}^{2}\right)$ and transforms to a vortex wall (b) identifiable by the characteristic dark-bright contrast. (c) and (d) show similar behaviour with opposite current direction resulting in a displacement in the opposite direction for the $750 \mathrm{~nm}$ wide wire $\left(j=2 \times 10^{12} \mathrm{~A} / \mathrm{m}^{2}\right)$.

means that the energetically lower lying wall type for this geometry is a vortex wall, similar to what has been observed for slightly different geometries in Permalloy. ${ }^{13,21}$ Interestingly, we do not observe any changes up to this high current density $\left(1 \times 10^{12} \mathrm{~A} / \mathrm{m}^{2}\right)$, which is slightly higher than the critical current density that we find in Permalloy for a nominally identical geometry $\left[j_{c}=8 \times 10^{11} \mathrm{~A} / \mathrm{m}^{2}\right.$ (Refs. 11 and 16)].

We have extended our investigation to other $\mathrm{CoFeB}$ wire widths and we have observed changes in the wall spin structure from transverse to vortex for the $750 \mathrm{~nm}$ wires as well as wall motion in this geometry at a current density of $2 \times 10^{12} \mathrm{~A} / \mathrm{m}^{2}$ [see Fig. 2(c) and 2(d)]. For the narrower wires, we do not observe any changes up to a breakdown current density of $2-5 \times 10^{12} \mathrm{~A} / \mathrm{m}^{2}$, indicating that for these geometries the transverse wall constitutes the lower energy spin structure. Furthermore, the critical current density increases from $1 \times 10^{12} \mathrm{~A} / \mathrm{m}^{2}$ for the $1500 \mathrm{~nm}$ to $2 \times 10^{12} \mathrm{~A} / \mathrm{m}^{2}$ for the $750 \mathrm{~nm}$ lines and to above $5 \times 10^{12} \mathrm{~A} / \mathrm{m}^{2}$ for the narrower lines. This indicates that for our samples the critical current density is governed by extrinsic pinning at edge defects, which increases in tandem with the critical field for domain wall motion for decreasing wire widths ${ }^{17}$ analogous to the inversely proportional scaling behavior known for field induced-domain wall motion.

This could also explain why we do not see a reduction in the critical current density for $\mathrm{CoFeB}$ compared to Permalloy, since it is the pinning at edge irregularities, which is present in $\mathrm{CoFeB}$ as well as in Permalloy and not the intrinsic pinning at grain boundaries (which would be absent in $\mathrm{CoFeB})$ that governs the $j_{c}$ in our samples.

To further study the origin of the high current density compared with Permalloy, we carried out magneto-optical Kerr effect measurements to study the field induced domain wall motion. For the $1500 \mathrm{~nm}$ wide and $20 \mathrm{~nm}$ thick CoFeB wires, we find a depinning field of $16.7 \pm 0.3 \mathrm{G}$ and for Permalloy with nominally the same geometry the depinning field is $13.4 \pm 0.6 \mathrm{G}$.

The coercivity (nucleation field) for $\mathrm{CoFeB}$ on the other hand is $30 \%$ smaller than in Permalloy. These facts support our interpretation that domain wall depinning either field or 

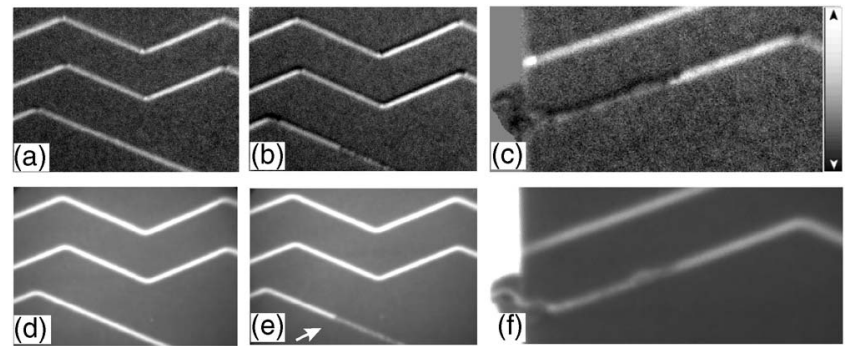

FIG. 3. The images in the top row show the magnetic contrast for the $400 \mathrm{~nm}$ wide wires and in the lower row the corresponding topographic images are presented. After applying a vertical magnetic field DWs are formed at the kinks (a). After pulse injection $\left(j=2 \times 10^{12} \mathrm{~A} / \mathrm{m}^{2}\right)$ the DWs are still located at the kinks (b), but the topographic image (e) reveals the structural damage of the wires, especially of the lower one (highlighted by an arrow). Images (c) and (f) show strong damage at the interface between the Au pad and the $\mathrm{CoFeB}$ wire where part of the Au has evaporated, but the DW in (c) is still unchanged located at the kink.

current-induced is dominated by extrinsic pinning at edge irregularities, whereas nucleation of domains is primarily governed by intrinsic pinning.

When we inject current pulses above $2 \times 10^{12} \mathrm{~A} / \mathrm{m}^{2}$, structural damage starts to set in. This is seen in Fig. 3 for the $400 \mathrm{~nm}$ wide wires where we present an image before and after a pulse injection. At the interface between the $\mathrm{Au}$ and the $\mathrm{CoFeB}$, the wires have been damaged, which is then also reflected in an increase of the resistance by $50 \%$, but the domain walls did not move.

Compared to the Permalloy wires, a key difference of the behavior of the $\mathrm{CoFeB}$ wires is that we do not observe any nucleation or annihilation of domain walls, which would indicate heating above the Curie temperature. This can be explained by the fact that the Curie temperature of $\mathrm{CoFeB}$ [>1300 K (Ref. 24)] is more than $450 \mathrm{~K}$ higher than that of Permalloy. This means that even for the highest current densities where structural damage, for instance due to electromigration occurs, the sample temperature stays below the Curie temperature.

\section{CONCLUSIONS}

In summary, we have imaged current-induced domain wall motion in $\mathrm{CoFeB}$ wires with varying widths. We observe transverse domain walls for wires up to $1500 \mathrm{~nm}$ in width, in agreement with the low saturation magnetization present in this material. High critical current densities of $j_{c}>1 \times 10^{12} \mathrm{~A} / \mathrm{m}^{2}$ are necessary to displace a domain wall in a $1500 \mathrm{~nm}$ wide wire. This critical current density increases as the wire width is reduced, which points to extrinsic defects as the main source of pinning and means that we do not observe any wall motion for wire widths $<750 \mathrm{~nm}$ for the current densities available. Earlier results on critical current densities found in $\mathrm{Co} / \mathrm{Cu} / \mathrm{CoFeB}$ multilayers are significantly smaller than our results. ${ }^{15}$ However, the results are difficult to compare directly since critical fields and currents tend to be smaller in multilayer structures.

In contrast to Permalloy structures, we find no random nucleation and annihilation of domain walls for current densities up to the breakdown density where structural damage sets in. This is attributed to the higher Curie temperature in $\mathrm{CoFeB}$ compared to Permalloy. From our measurements, we can conclude that in the material studied here, the domain wall pinning is primarily due to edge roughness and not due to pinning at intrinsic grain boundaries, which should be absent in $\mathrm{CoFeB}$.

\section{ACKNOWLEDGMENTS}

This work was supported by the Deutsche Forschungsgemeinschaft (SFB 513 and SPP1133), the Landesstiftung Baden-Württemberg, the Human Resources and Mobility programme (Marie Curie Actions), the Interreg III A program, and Samsung. Part of this work was performed at the Swiss Light Source, Paul Scherrer Institut, Villigen, Switzerland.

${ }^{1}$ S. S. P. Parkin, U.S. Patent No. 6,834,005 (September 11, 2005).

${ }^{2}$ D. A. Allwood, G. Xiong, M. D. Cooke, C. C. Faulkner, D. Atkinson, N. Vernier, and R. P. Cowburn, Science 296, 5575 (2002).

${ }^{3}$ L. Berger, Phys. Rev. B 54, 9353 (1996).

${ }^{4}$ J. C. Slonczewski, J. Magn. Magn. Mater. 159, L1 (1996).

${ }^{5}$ E. Martinez, L. Lopez-Diaz, O. Alejos, L. Torres, and C. Tristan, Phys. Rev. Lett. 98, 267202 (2007).

${ }^{6}$ A. Thiaville, Y. Nakatani, J. Miltat, and Y. Suzuki, Europhys. Lett. 69, 990 (2005).

${ }^{7}$ A. Yamaguchi, T. Ono, S. Nasu, K. Miyake, K. Mibu, and T. Shinjo, Phys. Rev. Lett. 92, 077205 (2004).

${ }^{8}$ J. Grollier, P. Boulenc, V. Cros, A. Hamzic, A. Vaurés, A. Fert, and A. Faini, Appl. Phys. Lett. 83, 509 (2003).

${ }^{9}$ N. Vernier, D. A. Allwood, D. Atkinson, M. D. Cooke, and R. P. Cowburn, Europhys. Lett. 65, 526 (2004).

${ }^{10}$ M. Kläui, C. A. F. Vaz, J. A. C. Bland, W. Wernsdorfer, G. Faini, E. Cambril, L. J. Heyderman, F. Nolting, and U. Rüdiger, Phys. Rev. Lett. 94, 106601 (2005).

${ }^{11}$ M. Kläui, M. Laufenberg, L. Heyne, U. Rüdiger, C. A. F. Vaz, J. A. C. Bland, L. C. Heyderman, S. Cherifi, A. Locatelli, T. O. Mentes, and L. Aballe, Appl. Phys. Lett. 88, 232507 (2006).

${ }^{12}$ A. Yamaguchi, T. Ono, S. Nasu, K. Miyake, K. Mibu, and T. Shinjo, Appl. Phys. Lett. 86, 012511 (2005).

${ }^{13}$ F. Junginger, M. Kläui, M. Laufenberg, L. Heyne, D. Backes, U. Rüdiger, C. A. F. Vaz, and J. A. C. Bland, Appl. Phys. Lett. 90, 132506 (2007).

${ }^{14}$ G. Tatara and H. Kohno, Phys. Rev. Lett. 92, 086601 (2004).

${ }^{15}$ S. Laribi, V. Cros, M. Munoz, J. Grollier, A. Hamzic, C. Deranlot, A. Fert, E. Martinez, L. Lopez-Diaz, L. Vila, G. Faini, S. Zoll, and R. Fournel, Appl. Phys. Lett. 90, 232505 (2007).

${ }^{16}$ L. Heyne and M. Kläui (unpublished).

${ }^{17}$ W. Y. Lee, H. T. Leung, W. Zhang, Y. B. Xu, A. Hirohata, C. C. Yao, B. C. Choi, D. G. Hasko, and J. A. C. Bland, IEEE Trans. Magn. 35, 3883 (1999).

${ }^{18}$ D. Backes, L. J. Heyderman, C. David, R. Schaublin, M. Kläui, H. Ehrke, U. Rüdiger, C. A. F. Vaz, J. A. C. Bland, T. Kasama, and R. E. DuninBorkowski, Microelectron. Eng. 83, 1726 (2006).

${ }^{19}$ S. U. Jen, Y. D. Yao, Y. T. Chen, J. M. Wu, C. C. Lee, T. L. Tsai, and Y. C. Chang, J. Appl. Phys. 99, 053701 (2006).

${ }^{20}$ J. Stöhr, Y. Wu, B. D. Hermsmeier, M. G. Samant, G. R. Harp, S. Koranda, D. Dunham, and B. P. Tonner, Science 259, 658 (1993).

${ }^{21}$ M. Laufenberg, D. Backes, W. Bührer, D. Bedau, M. Kläui, U. Rüdiger, C. A. F. Vaz, J. A. C. Bland, L. J. Heyderman, F. Nolting, S. Cherifi, A. Locatelli, R. Belkhou, S. Heun, and E. Bauer, Appl. Phys. Lett. 88, 052507 (2006).

${ }^{22}$ K. Yagami, A. A. Tulapurkar, A. Fukushima, and Y. Suzuki, Appl. Phys. Lett. 85, 5634 (2004).

${ }^{23}$ D. Backes, C. Schieback, M. Kläui, F. Junginger, H. Ehrke, P. Nielaba, U. Rüdiger, L. J. Heyderman, C. S. Chen, T. Kasama, R. E. DuninBorkowski, C. A. F. Vaz, and J. A. C. Bland, Appl. Phys. Lett. 91, 112502 (2007).

${ }^{24}$ K. Nagasaka, L. Varga, Y. Shimizu, S. Eguchi, and A. Tanaka, J. Appl. Phys. 87, 6433 (2000). 